\title{
Hongkong Observations of Eclipses and other Phenomena.
}

The following phenomena have been observed with the Lee Equatoreal, aperture six inches. The epochs are given in Hongkong Mean Time and the magnifying power is added.

Jupiter's Satellites.

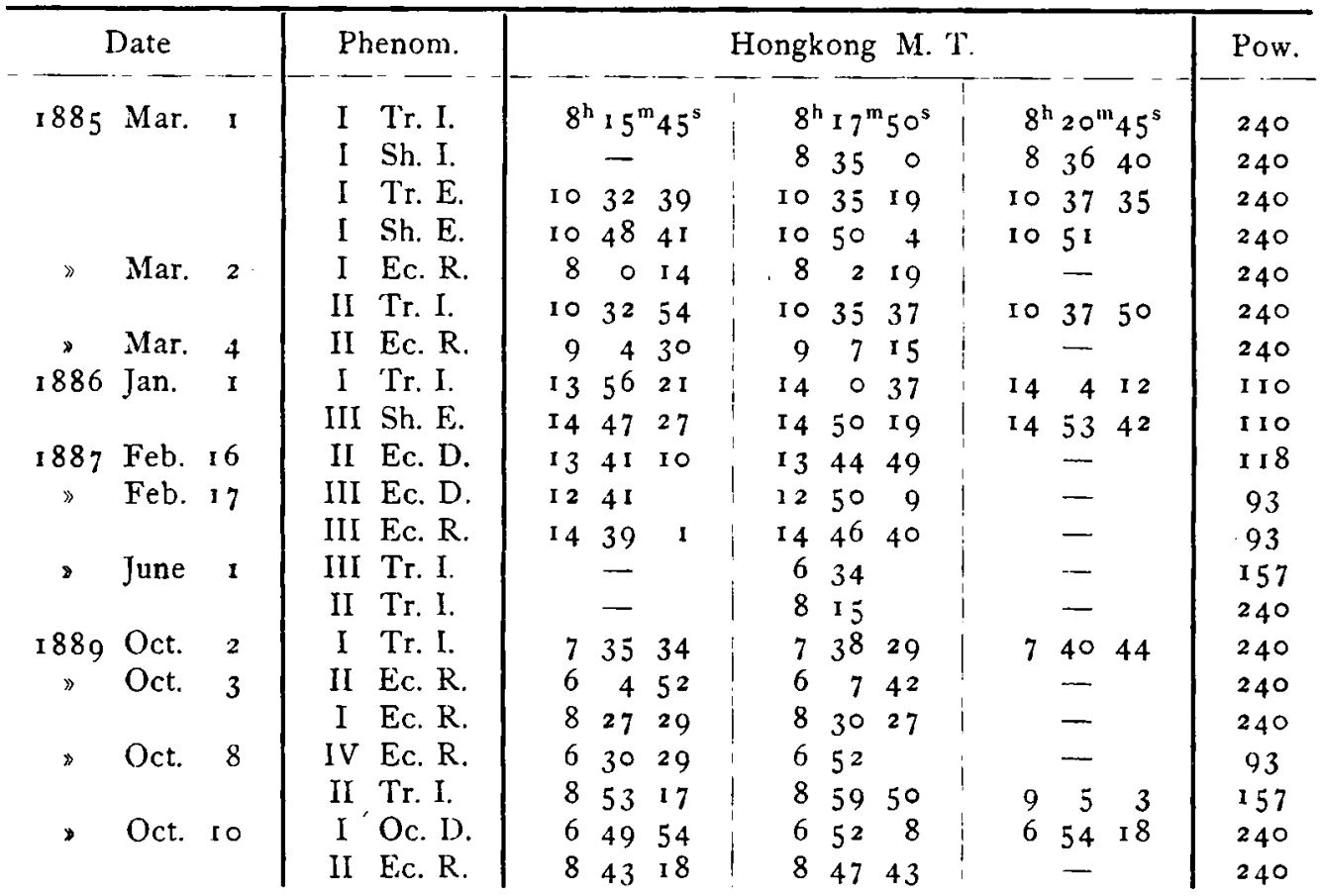

I 889 October 8 the fourth satellite ceased to increase in brightness at the time stated but remained dull the whole evening. The observation of the second satellite on the same date is uncertain owing to poor definition and the Ec. $R$. of the same on the $10^{\text {th }}$ is uncertain owing to haze.

A partial eclipse of the Sun was observed: 1887 Aug. $192^{\mathrm{h}} 55^{\mathrm{m}} 53^{\mathrm{s}}$ and an occultation of a star by the Moon: 1887 Oct. $25 \quad 6^{\mathrm{h}} 5^{6^{\mathrm{m}}} 3^{5.8}$.

An occultation of Jupiter and his satellites was observed on the $1^{\text {st }}$ October 1889 (Power 93). The Moon took a large fraction of a second to pass across the diameters of the satellites. At the moment of external contact Jupiter looked like a very elongated ellipse the largest diameter of which was parallel to the tangent to the Moon's limb.

\begin{tabular}{|c|c|c|c|c|c|c|}
\hline & IV & $6^{\mathrm{h}} 35^{\mathrm{I}}$ & $15 \cdot 5$ & Entrance : & 4 & $6^{\mathrm{h}} 49^{\mathrm{m}} 2 \mathrm{r}^{\mathrm{s}} \cdot 5$ \\
\hline & III & 646 & $5^{8.0}$ & & 24 & $\begin{array}{llll}6 & 5 & \text { I } & 10.0\end{array}$ \\
\hline & II & 647 & I.O & & & \\
\hline
\end{tabular}

Exit : II $8^{\mathrm{h}} 12^{\mathrm{m}} 10.0$

$$
\begin{array}{lllll}
4 & & & \\
4 & 8 & \text { I } 4 & 53.5
\end{array}
$$

W. Doberck.

\section{Ueber die Corona-Photographien 1887 Aug. 18-19.}

\section{Von A. Belopolsky.}

Ich habe einige Versuche gemacht, die gegenseitige Lage der schärfsten Ausläufer (Strahlen) auf den CoronaPhotographien von den Stationen: Petrowsk, Jurjewetz, Blagodatj, Krasnojarsk und Japan (Echigo) zu bestimmen und zu vergleichen. Ich mass nämlich die Positionswinkeldifferenz zwischen einer Protuberanz (Positionswinkel $143^{\circ}$, die in meinem Berichte über die Sonnenfinsterniss durch (5) be. zeichnet war, vergl. Ann. de Moscou) und jeder der CoronaAusläufer, die in meinem Berichte mit den Buchstaben: $A, B C, D, E, G, H$ und $M$ bezeichnet sind.

Diese Ausläufer sind auf den erwähnten Photographien alle leicht zu erkennen; der Unterschied, den man zwischen ihnen auf verschiedenen Photographien findet, liegt offenbar in der verschiedenen Schärfe der Bilder, was wahrscheinlich von den photographischen Apparaten, Expositionsdauer, Klarheit des Himmels etc. abhängt.

Die folgende Tabelle giebt die oben erwähnte Positionswinkeldifferenz. Die Messungen wurden mit einer Sicherheit von $1^{\circ}$ bis $5^{\circ}$, je nach der Schärfe der Begrenzung jedes Ausläufers, gemacht.

$\mathrm{Zu}$ besserer Orientirung lege ich dem Artikel eine schematische Zeichnung der Corona bei. 